\title{
Molecular alterations in gastric cancer and the surrounding intestinal metaplastic mucosa: an analysis of isolated glands
}

\author{
Ryo Sugimoto ${ }^{1}$ - Wataru Habano ${ }^{2} \cdot$ Naoki Yanagawa $^{1} \cdot$ Risaburo Akasaka $^{3} \cdot$ Yosuke Toya $^{3} \cdot$ Akira Sasaki $^{4}$. \\ Takayuki Matsumoto ${ }^{3} \cdot$ Tamotsu Sugai $^{1}$ (i)
}

Received: 7 September 2020 / Accepted: 11 October 2020 / Published online: 3 November 2020

(c) The International Gastric Cancer Association and The Japanese Gastric Cancer Association 2020

\begin{abstract}
Background Intestinal metaplasias (IMs) are generally regarded as pre-neoplastic gastric lesions. However, molecular alterations including genetic and epigenetic changes occurring in individual IM glands are not well defined.

Aims We sought to identify DNA methylation status, microsatellite instability (MSI) and allelic imbalance (AI) occurring in individual IM glands and non-IM glands within the same mucosa.

Methods We divided examined isolated gland obtained from GC into 4 components: isolated cancer, antral isolated intestinal metaplastic tissue, antral isolated non-metaplastic gland and isolated non-metaplastic gland derived from the greater curvature of the most distant gastric body without mucosal atrophy. We examined AI and microsatellite instability statuses using PCR-based microsatellite analysis. Next, the DNA methylation status (high methylation epigenome [HME], intermediate methylation epigenome [IME], and low methylation epigenome [LME]) was investigated. DNA methylation analysis of $C D K N 2 A$, mir34-b/c and $M L H I$ genes was also performed.

Results Although antral isolated IM glands were characterized by IME, isolated non-IM glands showed LME. In isolated cancer glands, HME was frequently found, compared with isolated non-IM glands. DNA methylation of mir34-b/c was common in isolated cancer and IM glands, whereas DNA methylation of $C D K N 2 A$ was a rare event in isolated samples. The MLHI gene was not methylated in isolated non-IM glands. Although multiple AIs were frequently found in isolated cancer glands, a few AIs were detected in isolated IM glands.

Conclusions We suggest that the DNA methylation status and the status of the mir34-b/c gene among isolated samples of IMs and isolated non-IM glands have an impact on IM development.
\end{abstract}

Keywords Crypt isolation method · DNA methylation · Intestinal metaplasia $\cdot$ Gastric cancer $\cdot$ Loss of heterozygosity

Electronic supplementary material The online version of this article (https://doi.org/10.1007/s10120-020-01130-z) contains supplementary material, which is available to authorized users.

Tamotsu Sugai

tsugai@iwate-med.ac.jp

1 Department of Molecular Diagnostic Pathology, School of Medicine, Iwate Medical University, 2-1-1, Shiwa,

Yahaba, Morioka 028-3695, Japan

2 Department of Pharmacodynamics and Molecular Genetics, School of Pharmacy, Iwate Medical University, 2-1-1, Shiwa, Yahaba, Morioka 028-3695, Japan

3 Division of Gastroenterology, Department of Internal Medicine, School of Medicine, Iwate Medical University, 2-1-1, Shiwa, Yahaba, Morioka 028-3695, Japan

4 Department of Surgery, School of Medicine, Iwate Medical University, 2-1-1, Shiwa, Yahaba, Morioka 028-3695, Japan

\section{Introduction}

Intestinal metaplasia (IM) is closely associated with the pathogenesis of gastric cancer (GC) [1]. Two contrasting theories have been proposed $[2,3]$. The pre-cancerous theory has been advocated by Western pathologists according to Correa's established pathway of gastric carcinogenesis [2, 3], which is supported by the finding that IM is frequently observed in the surrounding mucosa of GC $[1,2]$. On the other hand, the para-cancerous theory has been favored by Japanese pathologists [3], given that none of the pathologists could observe the direct budding of a cancerous gland from an intestinal metaplastic gland [4]. However, it is not clear which of the two theories of gastric carcinogenesis is correct. 
Both genetic and epigenetic alterations have recently emerged as crucial molecular events during the progression of GC [5-9]. Whereas genetic alteration gives rise to structural changes in the DNA, epigenetic alteration, including DNA methylation, alters transcription without structurally changing DNA sequences [7, 8]. Recent study has shown that epigenetic alteration plays a central role in early gastric tumorigenesis and that genetic alteration may be associated with progression of GC [7-9]. IM, which is considered an early lesion related to gastric carcinogenesis, is frequently found in the surrounding mucosa of GC $[1,2]$. Many studies of IM have previously shown that epigenetic alterations, including changes in DNA methylation, are frequently detected in GC as well as in the surrounding gastric mucosa [10-13]. By contrast, genetic change is thought to be a rare molecular event in IM, though contradictory data were reported [10]. Importantly, previous study has not shown that molecular alterations are acquired in individual intestinal metaplastic glands [10]. Based upon these considerations, it is important that molecular differences between intestinal metaplastic and non-metaplastic glands within the surrounding intestinal mucosa are identified. Currently, little is known regarding the difference occurring in intestinal metaplastic mucosa (IMM).

In the present study, we used a crypt isolation method to divide GC into 4 different components: isolated cancer glands, antral isolated intestinal metaplastic glands, antral non-intestinal metaplastic glands and isolated non-intestinal metaplastic glands, all of which were derived from the greater curvature of the most distant gastric body mucosa. We attempted to identify genetic and epigenetic alterations occurring in individual isolated glands in GC and the surrounding mucosa.

\section{Materials and methods}

\section{Patients}

We examined 24 GCs and the surrounding mucosa in the present study. Pathological diagnosis was made according to Japanese histological classification with a slight modification $[14,15]$. The pathologic factors analyzed included lymphovascular invasion, invasive tumor pattern and the grade of differentiation. Disease stage was determined using the TNM classification of the Japanese Gastric Cancer Association 7th edition [14]. No patients had undergone chemo- or radio-therapy prior to surgery. Patients with a family history or with a past medical history of gastric cancer were not included in this study. Detailed clinicopathological findings are shown in Table 1 .
Table 1 Clinicopathological findings of gastric cancer tissues sampled in this study

\begin{tabular}{ll}
\hline & Cases $(\%)$ \\
\hline Sex (man/woman) & 24 \\
Age (median) & $15 / 9$ \\
Locus & $56-87(70.5)$ \\
U & \\
M & $5(20.8)$ \\
L & $6(25.0)$ \\
Histology & $13(54.2)$ \\
WDA & \\
MDA & $10(41.7)$ \\
PAP & $12(50.0)$ \\
Stage & $2(8.7)$ \\
I & \\
II & $11(45.8)$ \\
III & $1(4.2)$ \\
IV & $8(33.3)$ \\
\hline
\end{tabular}

$W D A$ well-differentiated adenocarcinoma, $M D A$ moderately differentiated adenocarcinoma, $P A P$ papillary adenocarcinoma, $U$ upper body, $M$ middle body, $L$ lower body

\section{Crypt isolation technique}

Samples from tumors and from the normal surrounding gastric mucosa were obtained at the time of tumor resection. Tumor samples were obtained from the invasive front, whereas the normal surrounding mucosa was taken from the antral mucosa. In addition, the greater curvature of the most distant mucosa without mucosal atrophy was also obtained as a control for genetic analysis. Gland isolation was performed as previously described $[16,17]$. Briefly, fresh tumor samples and normal gastric mucosa were minced into small pieces using a razor, then incubated at $37{ }^{\circ} \mathrm{C}$ for $50 \mathrm{~min}$ in $\mathrm{Ca}^{++} / \mathrm{Mg}^{++}$-free Hanks balanced salt solution containing $30 \mathrm{mmol} / \mathrm{L}$ EDTA.

The isolated glands were fixed in $70 \%$ ethanol and stored at $4{ }^{\circ} \mathrm{C}$ until used for DNA extraction. The remaining isolated glands were fixed and paraffin-embedded tissue was made in accordance with routine histological procedures. Next, histological sections were examined by light microscopy to confirm the histopathological findings. There was no evidence of contamination of crypt cells by interstitial cells using this technique.

The surrounding non-neoplastic mucosa was isolated by following the steps described below. First, the glands were separated from the lamina propria mucosa. The isolated glands were stained with Alcian Blue at $\mathrm{pH} 2.5$. The isolated glands were divided into 2 components according to the presence/absence of goblet cells revealed by positive 
staining with Alcian Blue. As a result, isolated nonneoplastic glands were classified into 2 types: intestinal metaplastic glands that stained Alcian Blue-positive for goblet cells and non-intestinal metaplastic glands lacking staining. Consequently, the isolated glands from the antral mucosa were classified into 4 categories: isolated cancer glands, isolated intestinal metaplastic glands, isolated nonintestinal glands and isolated non-intestinal metaplastic glands that were obtained from the greater curvature of the most distant gastric body mucosa without atrophy. In the present study, 20-30 isolated cancer glands were obtained. In addition, 15-25 isolated metaplastic and non-metaplastic glands were collected from the same antral mucosa. Moreover, 20-25 isolated non-metaplastic glands were obtained from the distant greater curvature as a control.

\section{DNA extraction}

DNA was extracted from isolated normal and tumor tissues by sodium dodecyl sulfate (SDS) lysis and proteinase $\mathrm{K}$ digestion, followed by a phenol-chloroform procedure as reported previously [10].

\section{DNA methylation analysis}

The PyroMark Q24 system for pyrosequencing was used to assess the DNA methylation status of selected markers. Primer sequences were designed using Qiagen's Pyromark Assay Design 2.0 software. Detailed methods were described elsewhere $[18,19]$.

DNA methylation at the 6 specific promoters originally described by Yagi and colleagues was quantified. Methylation of 3 markers (RUNX3, MINT31 and LOX) was analyzed, and samples with at least 2 methylated markers were defined as having a highly methylated epigenotype (HME). The remaining tumors were also screened for methylation at 3 other markers (NEUROG1, ELMO1, and THBD), and they were defined as intermediate methylation epigenotype (IME) tumors if they had at least 2 methylated markers out of the 3 markers proposed as a second panel. Tumors not classified as HME or IME were designated as low methylation epigenotype (LME). In addition, the methylation status of CDKN2A (cyclin-dependent kinase inhibitor $2 A$ ), mir-34b/c and $M L H 1$ which are closely associated with gastric carcinogenesis were also quantified using the Qiagen's Pyromark Assay Design 2.0 software. The primer design used in this study was previously reported $[20,21]$.

\section{Analysis of MSI}

MSI status was determined by $5 \mathrm{NCI}$ markers, including BAT25, BAT26, D2S123, D5S346, and D17S250 [22]. MSI-high (MSI-H) was defined as 2 or more markers being unstable; MSI-low (MSI-L) was defined as 1 marker being unstable; and microsatellite stable (MSS) was defined as the absence of instability.

\section{Analysis of allelic imbalances at chromosomal loci}

DNA extracted from isolated glands (as described above) were analyzed for allelic imbalance by polymerase chain reaction (PCR) amplification using polymorphic dinucleotide repeat sequences, including 24 markers on chromosomes 1p (D1S228, D1S548 and D1S507), 3p (D3S2402 and D3S1234), 4p (D4S2639 and D4S1601), 5q (D5S107, D5S346, D5S82 and D5S299), 8q (D8S201, D8S513 and D8S532), 9p (D9S171 and D9S1118), 13q (D13S162); 17p (TP53), 18q (D18S487, D18S34 and DCC) and 22q (D22S274, D22S1140 and D22S1168) [7]. The sequences of these primers were obtained from The Genome Database (https://gdbwww.gdb.org/gdb/). PCR was performed using a DNA autosequencer (Applied Biosystems 9600 Sequencer; Applied Biosystems, Foster City, CA, USA), as previously described $[7,10]$. The data from the PCR analyses were collected automatically and analyzed using GeneScan software (Applied Biosystems) for allele scoring (allelic ratio) and assessment of AI, as described previously [7]. The formula employed for the calculation was T2:T1/N2:N1, where T1 and $\mathrm{N} 1$ were the height values for the smaller allele, and $\mathrm{T} 2$ and N2 were the height values for the larger allele of the tumor $(\mathrm{T})$ and normal $(\mathrm{N})$ samples, respectively. When AIs were observed in at least 1 locus of the chromosomal locus examined, the imbalances of those loci were confirmed. In this study, we defined AI as more than a 50\% difference in this ratio. When the result was a ratio of 0.50 or lower for the tumor sample the case was interpreted as an AI.

\section{Statistical analysis}

Data obtained for frequencies of methylation status and allelic imbalance based on each subgroup were analyzed using Fisher exact tests with the aid of JMP Pro 13.0 software (SAS Institute Inc., Cary, NC, USA). If statistical differences between the 4 groups were found, statistical analysis between two groups was further performed using chi-square tests (JMP Pro 13.0 software) with Bonferroni correction.

\section{Results}

Representative figures for isolated glands, their histological features and molecular analyses are shown in Fig. 1. Although $21 \mathrm{GCs}$ were available for genetic analysis, all non-neoplastic samples $(n=24)$ were available for genetic analysis. 

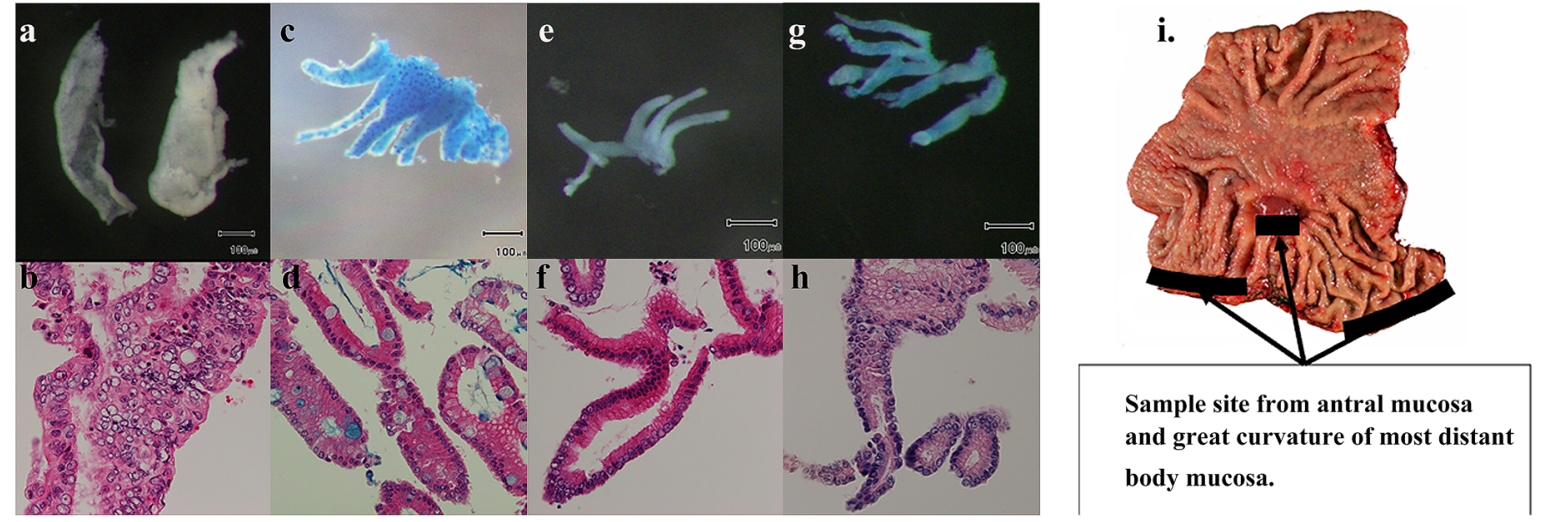

\section{j. DNA methylation analysis}

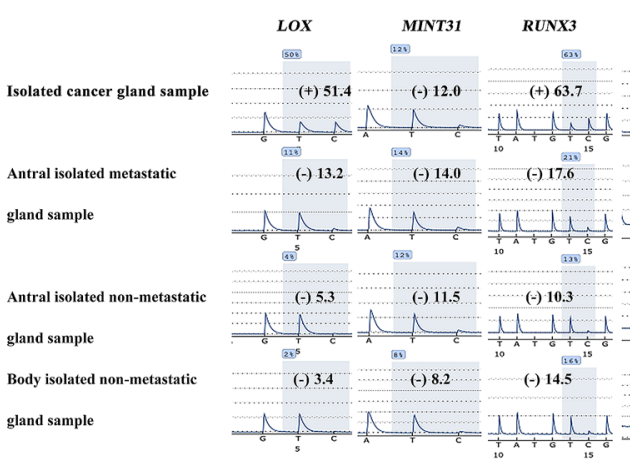

Fig. 1 Representative figure of isolated sample obtained from cancer, antral and most distant body mucosae. a Isolated cancer gland with negative staining of Alcian Blue. b Histological features of isolated cancer gland. c Antral isolated metaplastic gland with positive staining with Alcian Blue. d Histological features of isolated metaplastic gland. e Antral isolated non-metaplastic gland without staining of Alcian Blue. f Histological features of an antral isolated non-metaplastic gland $\mathbf{g}$ Body isolated non-metaplastic gland without staining with Alcian Blue; $\mathbf{h}$ Histological features of an isolated non-neoplas-

\section{k. Methylation status}

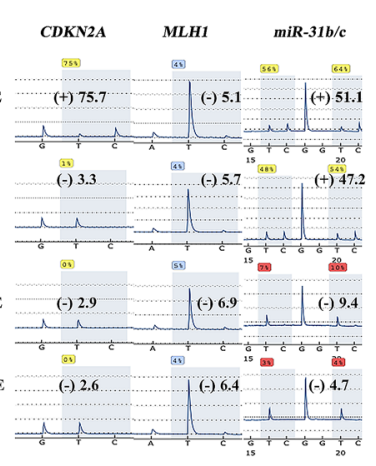

1. Allelic imbalance

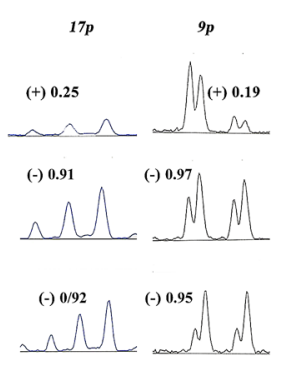

\section{Analysis of MSI status in each isolated gland}

Although MSI was found in 4 (19.0\%) of the 21 isolated cancer gland samples, no MSI was detected in the isolated antral metaplastic or the non-metaplastic gland samples, or isolated non-metaplastic glands obtained from the body mucosa without atrophy.

\section{Association of DNA methylation status with each isolated gland}

There were statistically significant differences in the frequency of HME, IME and LME among the 4 components according to multiple comparisons (Table 2). Additionally, 2 subgroup comparisons were added to identify statistically significant differences in the frequencies of HME, IME and LME between the 2 components. First, the frequency of HME was significantly higher in the isolated tic gland; i Sampling sites from antral mucosa and great curvature of distant body mucosa. j, k DNA methylation analysis. Isolated cancer glands showed HME, and antral isolated metaplastic glands showed IME, both antral and body isolated non-metaplastic glands indicate an LME (numerical data are methylation rate). 1 Although AIs at $9 \mathrm{p}$ and $17 \mathrm{p}$ were found in isolated cancer gland samples, no AI was detected in either sample of isolated non-metaplastic gland samples (numerical data are AI rate)

cancer gland samples than in the antral non-metaplastic and isolated non-metaplastic gland samples from the greater curvature of the most distant gastric body mucosa without atrophy $(p<0.05)$. Second, there was a significant difference in the frequencies of IME among antral isolated metaplastic gland samples and isolated cancer glands $(p<0.01)$, antral isolated non-metaplastic glands $(p<0.001)$ and body non-metaplastic isolated gland samples $(p<0.001)$. In addition, we noted a statistically significant difference in the frequencies of IME between isolated cancer glands and body isolated non-metaplastic gland samples $(p<0.05)$. Third, the frequency of LME was statistically significantly higher in antral isolated non-metaplastic gland samples than in isolated cancer glands and antral isolated non-metaplastic gland samples ( $p<0.05$ and $p<0.01$, respectively). Moreover, there were significant differences in the frequencies of LME among bodies isolated from non-neoplastic glands and cancer 
Table 2 Frequencies of DNA methylation status in isolated cancer, antral isolated metaplastic, antral isolated non-metastatic and body isolated non-metaplastic glands

\begin{tabular}{|c|c|c|c|c|c|c|}
\hline & $\begin{array}{c}\text { Pathologic } \\
\text { al stage }\end{array}$ & $\begin{array}{l}\text { Isolated } \\
\text { cancer } \\
\text { glands } \\
(\%)\end{array}$ & $\begin{array}{c}\text { Antral } \\
\text { isolated } \\
\text { metaplastic } \\
\text { glands (\%) }\end{array}$ & $\begin{array}{c}\text { Antral } \\
\text { isolated } \\
\text { non-meta- } \\
\text { plastic } \\
\text { glands (\%) }\end{array}$ & $\begin{array}{c}\text { Body } \\
\text { isolated } \\
\text { non-meta- } \\
\text { plastic } \\
\text { glands (\%) }\end{array}$ & p-value \\
\hline \multirow[t]{2}{*}{ Total } & & 21 & 24 & 24 & 24 & \\
\hline & & \multicolumn{4}{|c|}{ * } & \\
\hline \multirow[t]{5}{*}{ HME } & & $6(28.6)$ & $1(4.2)$ & $0(0)$ & $0(0)$ & $p<0.001$ \\
\hline & $\mathrm{I} / \mathrm{II}$ & $3(14.3)$ & $1(4.2)$ & $0(0)$ & $0(0)$ & \\
\hline & III/IV & $3(14.3)$ & $0(0)$ & $0(0)$ & $0(0)$ & \\
\hline & & \multicolumn{4}{|c|}{$*$ *** } & \\
\hline & & $*$ & $*$ & $* *$ & & \\
\hline \multirow[t]{4}{*}{ IME } & & $6(28.6)$ & $20(83.3)$ & $4(16.7)$ & $0(0)$ & $p<0.001$ \\
\hline & $\mathrm{I} / \mathrm{II}$ & $3(14.3)$ & $9(37.5)$ & $2(8.3)$ & $0(0)$ & \\
\hline & III/IV & $3(14.3)$ & $11(45.8)$ & $2(8.3)$ & $0(0)$ & \\
\hline & & & * & $\frac{* * *}{* * *}$ & & \\
\hline \multirow[t]{3}{*}{ LME } & & $9(42.9)$ & $3(12.5)$ & $20(83.3)$ & $24(100)$ & $p<0.001$ \\
\hline & $\mathrm{I} / \mathrm{II}$ & $3(14.3)$ & $2(8.3)$ & $10(41.7)$ & $12(50.0)$ & \\
\hline & III/IV & $6(28.6)$ & $1(4.2)$ & $10(41.7)$ & $12(50.0)$ & \\
\hline
\end{tabular}

$H M E$ high methylation epigenotype, IME intermediate methylation epigenotype, $L M E$ low methylation epigenotype, $I M$ intestinal metaplastic glands, N.S not significant

Fisher's exact test $* * * p<0.001, * * p<0.01, * p<0.05$

isolated glands and antral isolated metaplastic glands $(p<0.001)$. Detailed data regarding DNA methylation status are depicted in Table 2.

\section{DNA methylation levels of CDKN2A, mir34b/c and $M L H 1$ genes in individual isolated cancer glands}

There was no significant difference in the frequencies of the methylation levels of the $C D K N 2 A$ gene among the 4 subgroups. However, the mir34b/c gene was significantly more methylated in isolated cancer gland samples compared with antral and body isolated non-metaplastic gland samples
(Table 3, $p<0.01$ and $p<0.001$, respectively). In addition, there were statistically significant differences in the methylation frequencies of $\mathrm{mir} 34 \mathrm{~b} / \mathrm{c}$ among body isolated nonmetaplastic glands and cancer isolated glands $(p<0.001)$ and antral isolated metaplastic glands $(p<0.01)$. However, we observed no difference in the frequencies of methylation of the $\mathrm{mir} 34 \mathrm{~b} / \mathrm{c}$ gene between cancer-isolated glands and antral isolated metaplastic glands. Finally, we found statistically significant difference in the frequencies of $M L H 1$ methylation among the isolated cancer gland $(4 / 21,19.0 \%)$ samples and the remaining 3 samples $(p<0.05)$. The data are summarized in Table 3. 
Table 3 nnFrequencies of DNA methylation of $C D K N 2 A$, miR-34b/c and $M L H-1$ genes in isolated cancer, antral isolated metaplastic, antral isolated non-metastatic and body isolated non-metaplastic gland samples

\begin{tabular}{|c|c|c|c|c|c|c|}
\hline & $\begin{array}{l}\text { Pathological } \\
\text { stage }\end{array}$ & $\begin{array}{c}\text { Isolated } \\
\text { cancer gland } \\
(\%)\end{array}$ & $\begin{array}{c}\text { Antral } \\
\text { isolated } \\
\text { metaplastic } \\
\text { glands (\%) }\end{array}$ & $\begin{array}{c}\text { Antral } \\
\text { isolated non- } \\
\text { metaplastic } \\
\text { glands (\%) }\end{array}$ & $\begin{array}{c}\text { Body } \\
\text { isolated non- } \\
\text { metaplastic } \\
\text { glands }(\%)\end{array}$ & p-value \\
\hline Total & & 21 & 24 & 24 & 24 & \\
\hline \multirow[t]{4}{*}{$C D K N 2 A$} & & $1(4.8)$ & $1(4.2)$ & $1(4.2)$ & $0(0)$ & N.S. \\
\hline & I / II & $0(0)$ & $1(4.2)$ & $1(4.2)$ & $0(0)$ & \\
\hline & III / IV & $1(4.8)$ & $0(0)$ & $0(0)$ & $0(0)$ & \\
\hline & & \multicolumn{5}{|c|}{$\frac{* * *}{* *}$} \\
\hline \multirow[t]{3}{*}{$\mathrm{miR}-34 \mathrm{~b} / \mathrm{c}$} & & 13 (61.9) & $13 \overline{(54.1)}$ & $2(8.3)$ & $1(4.2)$ & $p<0.001$ \\
\hline & I / II & $7(33.3)$ & $7(29.2)$ & $1(4.2)$ & $1(4.2)$ & \\
\hline & III / IV & $6(28.6)$ & $6(25.0)$ & $1(4.2)$ & $0(0)$ & \\
\hline \multirow[t]{3}{*}{$M L H-1$} & & $4(19.0)$ & $0(0)$ & $0(0)$ & $0(0)$ & $p<0.05$ \\
\hline & $\mathrm{I} / \mathrm{II}$ & $3(14.3)$ & $0(0)$ & $0(0)$ & $0(0)$ & \\
\hline & III / IV & $1(4.8)$ & $0(0)$ & $0(0)$ & $0(0)$ & \\
\hline
\end{tabular}

Fisher's exact test $* * * p<0.001, * * p<0.01, * p<0.05$, N.S. not significant

\section{Comparison of allelic imbalances at each examined chromosomal locus among each isolated gland}

There were statistically significant differences in the frequencies of AIs at each chromosomal locus we examined among isolated cancer gland samples, antral isolated metaplastic glands and antral isolated non-metaplastic gland samples. Multiple AIs were frequently found in isolated cancer gland samples $(1 \mathrm{p}, 9 / 15,60.0 \%$; $3 \mathrm{p}, 13 / 18,72.2 \%$; 4p, 13/19, 68.4\%; 5q, 11/17, 64.7\%; 8p, 12/16, 75.0\%; 9q, 11/18, 61.1\%; 13q, 8/12, 66.7\%; 17p, 14/16, 87.5\%; 18q, 13/18, 72.2\%; 22q, 11/17, 64.7\%). However, low frequencies of AIs at $1 \mathrm{p}(1 / 18,5.6 \%), 3 \mathrm{p}(1 / 22,4.5 \%)$, $4 \mathrm{p}(3 / 22,13.6 \%), 5 \mathrm{q}(4 / 21,19.0 \%), 8 \mathrm{p}(3 / 21,14.3 \%), 9 \mathrm{p}$ $(0 / 20), 13 q(0 / 21), 17 p(0 / 21), 18 q(6 / 22,27.3 \%)$ and $22 q$ $(2 / 22,9.1 \%)$ were observed in antral isolated metaplastic gland samples. In addition, low frequencies of AIs at $1 \mathrm{p}$ $(3 / 19,15.8 \%), 3 p(0 / 21), 4 p(0 / 22), 5 q(2 / 22,9.1 \%), 8 p$ (0/22), 9p (0/20), 13q (0/21), 17p (1/21, 4.8\%), 18q (4/23, $17.4 \%)$ and $22 \mathrm{q}(1 / 22,4.5 \%)$ were also detected in antral non-metaplastic gland samples. There were statistically significant differences in the frequencies of each AI among isolated cancer glands, isolated metaplastic glands and isolated non-metaplastic glands. Detailed data are shown in Fig. 2.

\section{Comparison of examined markers in stages I/II and III/IV in gastric cancer}

We asked whether there were differences in the frequencies of AI at each chromosomal locus. We also characterized DNA methylation status and individual methylation levels occurring in mir34-b/c, CDKN2A and MLH-1 to assess the possible differences between stages I/II and III/IV. There was no difference in the frequencies of the above-mentioned markers between the 2 categories (Tables 2, 3 and supplementary Table 1).

Finally, supplementary Table 2 demonstrates the DNA methylation status, MSI, and AIs in the isolated glands from cancer, intestinal metaplastic mucosa, non-metaplastic antral mucosa, and non-metastatic body mucosa of each case. 
Fig. 2 Frequencies of allelic imbalance at each chromosomal locus in isolated cancer, antral isolated metaplastic and antral isolated non-metaplastic gland samples

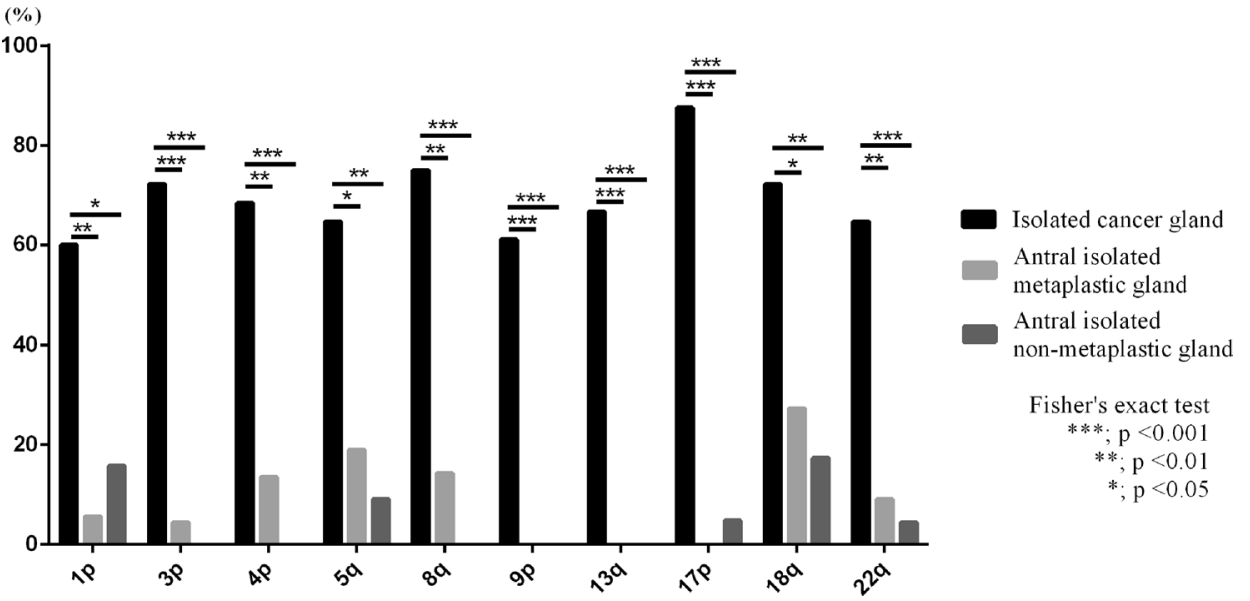

\section{Discussion}

Kaneda, et al. proposed that DNA methylation status could be classified into 3 categories: HME, IME and LME. Such classification is primarily used in colorectal cancer $[18,19]$. The intermediate-methylation epigenotype strongly correlates to the $K R A S$-mutation (+) in CRC. Accordingly, this two-panel method is thought to be useful to evaluate DNA methylation status of CRC $[18,19]$. Whether this method of analysis is applicable to gastric cancer and intestinal metaplastic glandular cells is uncertain. In our previous study, we successfully classified DNA methylation status occurring in GC into 3 subtypes: HME, IME and LME. In addition, GC with an MSI phenotype could be primarily assigned into HME. This finding seems to support the two-panel method proposed by Kaneda et al. and its suitability for classification of DNA methylation status of GC. However, it is still unclear whether this classification could be used in DNA methylation abnormalities occurring in intestinal metaplastic glands. Global DNA methylation occurring in isolated IM gland and non-IM gland will be examined in the near future.

In the present study, he most common DNA methylation status in isolated metaplastic glands was IME, which might shift cells from non-neoplastic toward neoplastic growth. Furthermore, DNA methylation status was found to be LME in non-metaplastic glands obtained from the greater curvature of the most distant gastric body mucosa without atrophy. The characterization of isolated metaplastic glands as IME may predict future status [20]. However, the finding that the most common DNA methylation status was LME in non-metaplastic glands obtained from antral IMM is very interesting. That is, the difference between isolated metaplastic and isolated non-metaplastic glands was epigenetic, i.e., DNA methylation. In addition, our result suggests that DNA methylation is facilitated by development of IM, which is closely associated with $H$. pylori infection $[23,24]$. That is, DNA methylation cannot accumulate in non-metaplastic glands, even if it is found in the same IM in which a metaplastic gland is involved. This finding may support the concept that IM is an irreversible lesion [2]. This observation is the first to demonstrate that epigenetic alterations occur in non-metaplastic glands. The current results can contribute to the understanding of gastric carcinogenesis.

There are increasing data that methylation of the mir34$b / c$ gene plays a major role in gastric carcinogenesis [20, 25]. In addition, DNA methylation of the $\mathrm{mir} 34-b / c$ gene in the surrounding mucosa can be an excellent predictive marker of GC risk [16, 20]. These findings may be supported by the discovery that $\mathrm{mir} 34-\mathrm{b} / \mathrm{c}$ is a target of p53 and that it cooperates in the control of cell proliferation [26]. In the present study, molecular alterations occurring in metaplastic glands are characterized by high DNA methylation of the mir34-b/c gene, also frequently found in GC, consistent with previous studies [20,25]. This finding suggests that DNA methylation of the mir34-b/c gene occurring in an individual metaplastic gland may accelerate the development of IM. This is supported by the finding that DNA methylation of the mir34-b/c gene is detected in a limited number of nonmetaplastic gland samples. Finally, ZIKI may be one of the target genes that are closely associated with development of IM [27]. However, whether miR-34b/c or ZIK1 methylation was more closely associated with IM remains unknown. Further investigation will be needed.

Epigenetic alterations in the $C D K N 2 A$ gene are variable and depend on the type of cancer [28, 29]. Thus, Zou et al. suggested that hypermethylation of the $p 16$ gene promoter may play an important role in the pathogenesis of gastric precancerous lesions and early gastric adenocarcinoma [28]. Lima et al. showed that $C D K N 2 A$ methylation was associated with the carcinogenic process in diffuse-type and intestinal-type carcinomas compared to non-neoplastic samples [29]. In addition, Matsusaka et al. demonstrated that H. pylori infection promoted $C D K N 2 A$ DNA methylation at levels ranging from $21.3 \%$ to $45.0 \%$, correlating with poor 
tumor differentiation, increased lymph node metastasis, and lower survival rates of gastric cancer patients [30]. According to these studies, DNA methylation of the CDKN2A gene seems to be closely associated with gastric tumorigenesis. In the present study, however, methylation of the $C D K N 2 A$ gene plays a minor role in cancer glands and the surrounding metaplastic glands. There was a difference in the methylation status found in the present study and in previous reports. However, we suggest that the current results may be more reliable for analysis of the methylation of the $C D K N 2 A$ gene given that the target cell was morphologically determined by use of isolated glands.

In the present study, GC with an MSI in which the MLHI gene was methylated was detected in isolated cancer gland samples. However, MSI and DNA methylation of the $M L H I$ gene was not observed in isolated non-neoplastic samples obtained from surrounding mucosa of GC with an MSI phenotype. Previous studies have shown that $M L H 1$ is heavily methylated and consequently causes MSI in IM [31, 32]. The reason for the discrepancy in DNA methylation of the $M L H 1$ gene between previous studies and the present result remains unknown. However, the current result is believed to be a reliable and reproducible finding, given that isolated glands without interstitial cells were used. Therefore, we suggest that $M L H 1$ methylation does not occur in pre-cancerous lesions.

Previous studies have shown that multiple AIs drive progression of GC and other cancers [7,33]. Multiple AIs may be a biological marker to predict progression risk in GC [7, 8]. In the present study, although multiple AIs had accumulated in isolated cancer glands, a low frequency of AIs was found in non-neoplastic glands, including metaplastic and non-metaplastic glands. Based on this finding, accumulating AIs may play a minor role in the development of IM, though contradictory data were suggested in a previous publication [10]. This finding may suggest that irreversible change does not occur in metaplastic glands within the IM.

There are some limitations to this study. First, genomewide analyses of genetic and epigenetic status that are performed for comprehensive evaluation [9] were not used in the present study. An amount and quality of DNA sufficient for comprehensive genetic and epigenetic analyses could not be obtained in isolated metaplastic and non-metaplastic tissue within the same intestinal metaplastic mucosa. For that reason, we considered that PCR-based analysis of genetic and epigenetic alterations was suitable for isolated metaplastic and non-metaplastic glands. Second, this study might be small. However, it is difficult to obtain isolated metaplastic and isolated non-metaplastic glands, separately, within the same IMM. We suggest that the molecular differences between isolated metaplastic and non-metaplastic gland samples could be identified in the present study. In addition, we limited the target lesion to differentiated-type GC, given that isolation of poorly differentiated type adenocarcinoma, including signet ring cell carcinoma, is difficult. Further studies will be performed in the near future.

In conclusion, we divided a given GC sample into 4 components, including isolated cancer glands, antral isolated metaplastic glands, antral isolated non-metaplastic glands and isolated non-metaplastic glands obtained from the greater curvature of the most distant body mucosa. We showed differences in the molecular alterations (including DNA methylation) between isolated metaplastic gland samples and isolated non-metaplastic gland samples found within the same IMM. This result is significant because heterogeneous DNA methylation was found even in the same IMM. In addition, the present finding showed that DNA methylation of the mir34-b/c gene characterizes intestinal metaplastic glands. Finally, IMM may be stable in terms of genomic changes, given that multiple AIs did not accumulate in intestinal metaplastic glands. Further study will be pursued in the near future.

Acknowledgements We gratefully acknowledge the technical assistance of Ms. E. Sugawara and Mr. T. Kasai. We also thank members of the Department of Molecular Diagnostic Pathology, Iwate Medical University for their support.

Author contributions R. Sugimoto, who is the first author, constructed the Figures and Tables and performed statistical analysis. T. Sugai, who is the corresponding author, contributed to the preparation of the manuscript, including all aspects of data collection analysis and study design. N. Yanagawa helped pathological assessment. M. Akasaka, R, Y. Toya, and T. Matsumoto assisted with obtaining clinical data. A Sasaki contributed to interpretation of the surgical findings.

Funding None.

\section{Compliance with ethical standards}

Conflict of interest The authors declare that they have no conflict of interest associated with this manuscript.

Human rights statement and informed consent All procedures were in accordance with the ethical standards of Iwate Medical University and the Declaration of Helsinki. The study was approved by the institutional review board of Iwate Medical University, and written informed consent was obtained from all patients, as follows: This study was approved by the institutional review board of Iwate Medical University ("Molecular analyses of Gastric cancer and the surrounding mucosa using isolated cancer gland, isolated metaplastic and non-metaplastic glands"; approval number HGH29-11).

Informed consent Informed consent from patients was waived, but information on this study was disclosed in the study website during data collection. 


\section{References}

1. Correa P, Piazuelo MB, Wilson KT. Pathology of gastric intestinal metaplasia: clinical implications. Am J Gastroenterol. 2010;105:493-8. https://doi.org/10.1038/ajg.2009.728.

2. Walker MM. 1 Is intestinal metaplasia of the stomach reversible? Is intestinal metaplasia of the stomach reversible? Gut. 2003;52:1-4. https://doi.org/10.1136/gut.52.1.1.

3. Rugge M, Farinati F, Baffa R, Sonego F, Di Mario F, Leandro G, et al. Gastric epithelial dysplasia in the natural history of gastric cancer: a multicenter prospective follow-up study. Interdisciplinary Group on Gastric Epithelial Dysplasia. Gastroenterology. 1994;107:1288-96. https://doi.org/10.1016/0016-5085(94)90529 -0 .

4. Sugai T, Inomata M, Uesugi N, Jiao Y-F, Endoh M, Orii S, et al. Analysis of mucin, p53 protein and Ki-67 expressions in gastric differentiated-type intramucosal neoplastic lesions obtained from endoscopic mucosal resection samples: A proposal for a new classification of intramucosal neoplastic lesions based on nuclear atypia. Pathol Int. 2004;54:425-35.

5. Zaky AH, Watari J, Tanabe H, Sato R, Moriichi K, Tanaka A, et al. Clinicopathologic implications of genetic instability in intestinal-type gastric cancer and intestinal metaplasia as a precancerous lesion: proof of field cancerization in the stomach. Am J Clin Pathol. 2008;129:613-21. https://doi.org/10.1309/DFLEL PGPNV5LK6B1.

6. Huang RJ, Ende AR, Singla A, Higa JT, Choi AY, Lee AB, et al. Prevalence, risk factors, and surveillance patterns for gastric intestinal metaplasia among patients undergoing upper endoscopy with biopsy. Gastrointest Endosc. 2020;91(70-77):e1. https://doi. org/10.1016/j.gie.2019.07.038.

7. Sugai T, Sugimoto R, Habano W, Endoh M, Eizuka M, Tsuchida $\mathrm{K}$, et al. Genetic differences stratified by PCR-based microsatellite analysis in gastric intramucosal neoplasia. Gastric Cancer. 2017;20:286-96. https://doi.org/10.1007/s10120-016-0616-2.

8. Sugai T, Eizuka M, Arakawa N, Osakabe M, Habano W, Fujita $\mathrm{Y}$, et al. Molecular profiling and comprehensive genome-wide analysis of somatic copy number alterations in gastric intramucosal neoplasias based on microsatellite status. Gastric Cancer. 2018;21:765-75. https://doi.org/10.1007/s10120-018-0810-5.

9. Cancer Genome Atlas Research Network. Comprehensive molecular characterization of gastric adenocarcinoma. Nature. 2014;513:202-9. https://doi.org/10.1038/nature13480.

10. Sugai T, Habano W, Jiao YF, Toyota M, Suzuki H, Tsukahara M, et al. Molecular analysis of single isolated glands in gastric cancers and their surrounding gastric intestinal metaplastic mucosa. Oncol Rep. 2010;23(1):25-33.

11. Kang GH, Lee HJ, Hwang KS, Lee S, Kim JH, Kim JS. Aberrant $\mathrm{CpG}$ island hypermethylation of chronic gastritis, in relation to aging, gender, intestinal metaplasia, and chronic inflammation. Am J Pathol. 2003;163:1551-6. https://doi.org/10.1016/S0002 -9440(10)63511-0.

12. Calcagno DQ, Wisnieski F, Mota ERDS, Maia de Sousa SB, Costa da Silva JM, Leal MF, et al. Role of histone acetylation in gastric cancer: implications of dietetic compounds and clinical perspectives. Epigenomics. 2019;11:349-362. https://doi.org/10.2217/ epi-2018-0081.

13. Perri F, Cotugno R, Piepoli A, Merla A, Quitadamo M, Gentile A, et al. Aberrant DNA methylation in non-neoplastic gastric mucosa of $H$. pylori infected patients and effect of eradication. Am J Gastroenterol. 2007;102:1361-71. https://doi.org/10.111 1/j.1572-0241.2007.01284.x.

14. Japanese Gastric Cancer Association: Japanese classification of gastric carcinoma: 3rd English edition. Gastric Cancer 2011; $14: 101-112$.
15. Kushima R, Lauwers GY, Rugge M. Gastric dysplasia: WHO classification of tumours of the digestive system. Lyon: International Agency for Research on Cancer; 2019. p. 71-5.

16. Takahashi Y, Sugai T, Habano W, Ishida K, Eizuka M, Otsuka $\mathrm{K}$, et al. Molecular differences in the microsatellite stable phenotype between left-sided and right-sided colorectal cancer. Int J Cancer. 2016;139:2493-501. https://doi.org/10.1002/ijc.30377

17. Habano W, Sugai T, Nakamura S, Yoshida T. A novel method for gene analysis of colorectal carcinomas using a crypt isolation technique. Lab Invest. 1996;74:933-40.

18. Yagi K, Takahashi H, Akagi K, Matsusaka K, Seto Y, Aburatani $\mathrm{H}$, et al. Intermediate methylation epigenotype and its correlation to KRAS mutation in conventional colorectal adenoma. Am J Pathol. 2012;180:616-25. https://doi.org/10.1016/j.ajpat h.2011.10.010.

19. Kaneda A, Yagi. Two groups of DNA methylation markers to classify colorectal cancer into three epigenotypes. Cancer Sci 2011; 102: 18-24. https://doi.org/10.1111/j.1349-7006.2010.01712.x.

20. Suzuki H, Yamamoto E, Nojima M, Kai M, Yamano HO, Yoshikawa $\mathrm{K}$, et al. Methylation-associated silencing of microRNA$34 \mathrm{~b} / \mathrm{c}$ in gastric cancer and its involvement in an epigenetic field defect. Carcinogenesis. 2010;31:2066-73. https://doi.org/10.1093/ carcin/bgq203.

21. Aoki H, Yamamoto E, Yamano HO, Sugai T, Kimura T, Tanaka Y, et al. Subtypes of the Type II pit pattern reflect distinct molecular subclasses in the serrated neoplastic pathway. Dig Dis Sci. 2018;63:1920-8. https://doi.org/10.1007/s10620-018-5016-5.

22. Boland CR, Thibodeau SN, Hamilton SR, Sidransky D, Eshleman JR, Burt RW, et al. A National Cancer Institute Workshop on Microsatellite Instability for cancer detection and familial predisposition: development of international criteria for the determination of microsatellite instability in colorectal cancer. Cancer Res. 1998;58:5248-57.

23. Huang KK, Ramnarayanan K, Zhu F, Srivastava S, Xu C, Tan ALK, et al. Genomic and epigenomic profiling of high-risk intestinal metaplasia reveals molecular determinants of progression to gastric cancer. Cancer Cell. 2018;33(137-150):e5. https://doi. org/10.1016/j.ccell.2017.11.018.

24. Valenzuela MA, Canales J, Corvalán AH, Quest AF. Helicobacter pylori-induced inflammation and epigenetic changes during gastric carcinogenesis. World J Gastroenterol. 2015;21:12742-56. https://doi.org/10.3748/wjg.v21.i45.12742.n

25. Suzuki R, Yamamoto E, Nojima M, Maruyama R, Yamano HO, Yoshikawa K, et al. Aberrant methylation of microRNA-34b/c is a predictive marker of metachronous gastric cancer risk. $\mathrm{J}$ Gastroenterol. 2014;49:1135-44. https://doi.org/10.1007/s0053 5-013-0861-7.n

26. Corney DC, Flesken-Nikitin A, Godwin AK, Wang W, Nikitin AY. MicroRNA-34b and MicroRNA-34c are targets of p53 and cooperate in control of cell proliferation and adhesionindependent growth. Cancer Res. 2007;67:8433-8. https://doi. org/10.1158/0008-5472.CAN-07-1585.n

27. Mihara M, Yoshida Y, Tsukamoto T, Inada K, Nakanishi Y, Yagi $\mathrm{Y}$, et al. Methylation of multiple genes in gastric glands with intestinal metaplasia: A disorder with polyclonal origins. Am J Pathol. 2006;169:1643-51. https://doi.org/10.2353/ajpath.2006.060552.

28. Zou XP, Zhang B, Zhang XQ, Chen M, Cao J, Liu WJ. Promoter hypermethylation of multiple genes in early gastric adenocarcinoma and precancerous lesions. Hum Pathol. 2009;40:1534-42. https://doi.org/10.1016/j.humpath.2009.01.029.

29. Lima EM, Leal MF, Burbano RR, Khayat AS, Assumpção PP Bello MJ, et al. Casartelli C. Methylation status of ANAPC1, CDKN2A and TP53 promoter genes in individuals with gastric cancer. Braz J Med Biol Res. 2008;41:539-43. https://doi. org/10.1590/s0100-879x2008000600017. 
30. Matsusaka K, Funata S, Fukayama M, Kaneda A. DNA methylation in gastric cancer, related to Helicobacter pylori and EpsteinBarr virus. World J Gastroenterol. 2014;20:3916-26. https://doi. org/10.3748/wjg.v20.i14.3916.

31. Leung WK, Kim JJ, Kim JG, Graham DY, Sepulveda AR. Microsatellite instability in gastric intestinal metaplasia in patients with and without gastric cancer. Am J Pathol. 2000;156:537-43. https ://doi.org/10.1016/S0002-9440(10)64758-X.

32. Watari J, Moriichi K, Tanabe H, Kashima S, Nomura Y, Fujiya $\mathrm{M}$, et al. Biomarkers predicting development of metachronous gastric cancer after endoscopic resection: an analysis of molecular pathology of Helicobacter pylori eradication. Int $\mathrm{J}$ Cancer. 2012;130:2349-58. https://doi.org/10.1002/ijc.26275.

33. Sugai T, Eizuka M, Takahashi Y, Fukagawa T, Habano W, Yamamoto E, et al. Molecular subtypes of colorectal cancers determined by PCR-based analysis. Cancer Sci. 2017;108:427-34.

Publisher's Note Springer Nature remains neutral with regard to jurisdictional claims in published maps and institutional affiliations. 